\title{
Efficacy of Bladder Neck Incision (BNI) Versus Transurethral Resection of Prostate (TURP) in Management of Benign Prostatic Hyperplasia (BPH) Causing Obstruction: A Randomised Controlled Study
}

\author{
Hussein H. S. Saddam, Jain Sudhir Kumar, Singh Chandra Bhushan \\ General Surgery, Maulana Azad Medical College, New Delhi, India \\ Email: saddamhussainhs@gmail.com
}

How to cite this paper: Saddam, H.H.S., Jain Sudhir Kumar and Chandra Bhushan, S. (2019) Efficacy of Bladder Neck Incision (BNI) Versus Transurethral Resection of Prostate (TURP) in Management of Benign Prostatic Hyperplasia (BPH) Causing Obstruction: A Randomised Controlled Study. Open Journal of Urology, 9, 119-129.

https://doi.org/10.4236/oju.2019.98015

Received: July 3, 2019

Accepted: August 23, 2019

Published: August 26, 2019

Copyright $\odot 2019$ by author(s) and Scientific Research Publishing Inc. This work is licensed under the Creative Commons Attribution International License (CC BY 4.0).

http://creativecommons.org/licenses/by/4.0/

\begin{abstract}
Objective: To compare the efficacy of bladder neck incision (BNI) with transurethral resection of prostate (TURP) in the treatment of patients with urinary obstruction caused by benign prostatic hyperplasia (BPH) on the basis of short term follow up of 4 months. Patient and Methods: The study was conducted in Department of General Surgery in Maulana Azad Medical College, New Delhi. 60 men with proven clinical diagnosis of BPH of size 30 grams and less presenting with symptoms of bladder outlet obstruction (BOO) were randomised prospectively to undergo either of the two operative modalities. Preoperatively size of the prostate, symptom scoring (IPSS), peak flow rate $\left(\mathrm{Q}_{\max }\right)$ were assessed. Postoperatively and during 4 months follow up the following data were collected-operative time, catheterisation period, hospital stay, blood loss, $\mathrm{Q}_{\max }$ and IPSS. Results: Preoperative parameters in both the groups showed no statistically significant differences with respect to prostate size, $\mathrm{Q}_{\max }$ and IPSS Scoring. At 4 months follow up $\mathrm{Q}_{\max }$ increased from $(6.35 \pm 4.49)$ to $(16.41 \pm 2.28)$ in TURP group and $(4.51 \pm 3.57)$ to $(15.95 \pm 2.58)$ in BNI group. IPSS decreased from 18.70 to 5.7 in TURP group and 18.90 to 6.00 in BNI group. All differences were statistically significant. There was a statistically significant difference in operative time, blood loss, hospital stay, catheterisation timing favouring BNI. Conclusion: TURP and $\mathrm{BNI}$ are equally effective in providing symptomatic improvement. BNI has an upper hand in reference to operative time, hospital stay, duration of catheterisation and blood loss.
\end{abstract}




\section{Keywords}

Benign Prostatic Hyperplasia (BPH), Bladder Neck Incision (BNI), Bladder Outlet Obstruction (BOO), Peak Urinary Flow Rate $\left(\mathrm{Q}_{\max }\right)$, International Prostate Scoring System (IPSS)

\section{Introduction}

Surgical treatment of BPH is classically by TURP and is the gold standard in treatment of symptomatic BPH which has withstood the test of time [1]. However, in the last two decades, the role of TURP as gold standard therapy for patients with LUTS due to BPH has increasingly been challenged by the development of medical ( 5 alpha reductase inhibitors, alpha 1 receptor blockers) and less invasive interventional alternatives. The main driving force behind this development was a high prevalence of disease and importantly the apparently unchanged morbidity of TURP [2].

Transurethral incision of prostate (TUIP) or Bladder neck incision (BNI) is an endoscopic surgical procedure which is relatively simple, quick and technically easier. It has been asserted that BNI is an underused procedure [3] and at least half of the patients who currently undergo TURP for small BPH causing obstruction can be treated effectively with BNI thereby avoiding many of the risks of the former procedure [4]. However, it did not gain popularity due to emerging techniques like HoLEP.

The TUIP was first described by Botini in 1887 but it was revisited and popularised by Orandi in 1973 [4]. TUIP has shown to be less invasive than TURP with minimal complications.

There is a general consensus that a patient whose estimated prostate gland is $30 \mathrm{~g}$ or less, is an ideal candidate for BNI [5]. Therefore, there is a need to compare the results of BNI as a treatment modality with that of TURP in patients will small prostate having severe obstructive symptoms.

\section{Aims and Objectives}

To compare bladder neck incision to transurethral resection of the prostate in the management of benign prostatic hyperplasia with reference to:

1) Efficacy in relieving obstructive symptoms in terms of peak urinary flow rates and IPSS scoring being the primary objective.

The secondary objectives being

1) Operative time

2) Duration of hospital stay

\section{Materials and Methods}

From October 2016 to April 2018, patients presenting to Department of General Surgery, Maulana Azad Medical College and Lok Nayak Hospital, New Delhi with benign enlargement of the prostate with obstructive symptoms (hesitancy, 
poor or intermittent stream, straining, prolonged micturition, feeling of incomplete bladder emptying, dribbling) were evaluated and scored according to IPSS, while objective evidence of urinary tract obstruction was assessed by uroflowmetry and ultrasound KUBP with PVRU measurement. Those who fulfilled inclusion criteria were included in the study. They were assigned randomly using computer generated random number table to undergo either TUIP OR TURP.

Inclusion criteria:

Patients with $\mathrm{BPH}$ and prostatomegaly of up to $30 \mathrm{cc}$ with objective evidence of BOO shown by any of the following

1) Peak flow rate $<10 \mathrm{ml} / \mathrm{s}$. Patients with flow rate between 10 and $15 \mathrm{ml} / \mathrm{s}$ were included only after failure of 12 weeks of alpha blocker treatment.

2) Post void residual urine $>100 \mathrm{ml}$.

3) IPSS score $>18$. Patients with IPSS score of 7 to 18 were included only after failure of 12 weeks of alpha blocker treatment.

Exclusion criteria:

1) Suspected malignancy on DRE, USG or serum PSA.

2) Presence of bladder stones or bladder diverticulum at bladder neck.

3) Previous urethroplasty for strictures or hypospadiasis or existing stricture.

4) Those with prostate size on clinical assessment is more than grade II or more than 2 cystoscopic fields.

Sample size:

$$
n=\frac{\left(\sigma_{1}+\sigma_{2}\right)^{2}\left(\zeta_{\alpha 1-\alpha 2}+\zeta_{1-\beta}\right)^{2}}{\left(m_{1}-m_{2}\right)^{2}}
$$

where:

- $\zeta_{\alpha 1-\alpha 2}=1.96$ (at $95 \%$ confidence)

- $\zeta_{1-\beta}=0.84$ (at $80 \%$ power)

- $m_{1}=$ mean PFR in group $1(\mathrm{BNI}) 16.6$

- $m_{2}=$ mean PFR in group 2 (TURP) 18.4

- $\sigma_{1}=\mathrm{SD}$ of PFR in BNI 0.8

- $\sigma_{2}=$ SD of PFR in TURP 1

The values are derived from mean and standard deviation outcomes of previous study [6]. According to these values the sample size is 7.84 in each group and we took 30 patients in each group.

Randomisation:

Patients were randomised into two groups using computer generated random numbers which were kept in 60 sealed envelopes. Envelopes were opened just before the start of surgery by paramedics not involved in surgery.

Out of two groups, Group A underwent TURP while Group B underwent Bladder neck incision, BNI.

Methodology:

Informed written consent was taken from all the patients before enrolment to participate in the study. Patients had the option to opt out of the study at any point of time without compromising right of treatment. Complete clinical history in- 
cluding smoking, neurological symptoms, medication history and comorbidities were asked for. Physical examination along with DRE and IPSS scoring was done. They were also evaluated clinically for symptoms arising out of neurogenic bladder.

Baseline investigations were carried out including complete blood count, urine analysis and culture, blood sugar, urea, creatinine, serum electrolytes, investigations for anesthesia fitness and serum PSA. Uroflowmetry was done for each patient to find out peak and average flow rate for a voided volume of more than 200 $\mathrm{ml}$ on 2 occasions. Peak flow rate $<10 \mathrm{ml} / \mathrm{s}$ was taken as a sign of obstruction and those with peak flow rate lying in between 10 and $15 \mathrm{ml} / \mathrm{s}$ were treated with alpha blocker Tamsulosin for 12 weeks before deciding about their eligibility for this study. USG KUBP and TRUS done for each patient to estimate the size of prostate and PVRU. Preliminary urethrocystoscopic evaluations were made in every case to rule out any concomitant bladder pathology and to grade prostate enlargement.

A $26 \mathrm{Fr}$ continuous flow Karl Storz resectoscope was used in all procedures under spinal regional anesthesia. 1.5\% Glycine was used as irrigation fluid. At the end of procedure, a 22F 3 way Foley catheter was inserted and kept in bladder, balloon was inflated with $30 \mathrm{ml}$ of saline and connected to a closed drainage system after application of traction.

In TURP group, resection was done circumferentially up to anatomical capsule of the prostate, using Nesbit technique. First, the middle lobe was resected and an excavation between 5 and 7 o'clock up to the surgical capsule was formed. Successively, after middle lobe, the lateral lobes and then ventral tissues of the gland were resected. The apical parts of the gland are resected last. Extreme care was taken to preserve verumontanum and not go beyond it.

In TUIP group, two deep incisions at 5 and 7 o'clock position were made using Collin's knife. Incision was made from the trigone below the ureteric orifices, cutting the bladder neck and prostate to the sides of proximal end of verumontanum. Incisions were deepened till the fibres of prostatic capsules were seen.

Record of the time taken for surgery was noted. Postoperatively, continuous bladder wash with normal saline was continued as long as washout was blood stained. In every patient, total operating time, amount of irrigation fluid used in litres and number of packed cell volumes (in bags) transfusions required was recorded. Postoperative catheterisation period was noted. Every patient was followed up in surgical OPD at 1 week, 1 month and at 4 months post operatively. In follow up, patients were studied for subjective evaluation of outcome of operation, uroflowmetry and detailed symptom score (IPSS [7]).

All the data were recorded in predesigned data collection sheets and subjected to statistical analysis. Comparison of efficiency of both the procedures in relieving symptoms and advantages of TUIP over TURP were observed.

Parameters for comparison:

1) Duration of surgery

2) Duration of hospital stay

3) Persistence of symptoms at 4 months

4) Efficacy in relieving symptoms 
The process of randomization, allocation, follow-up and analysis of data of the study was done as shown in the Consort diagram (Figure 1).

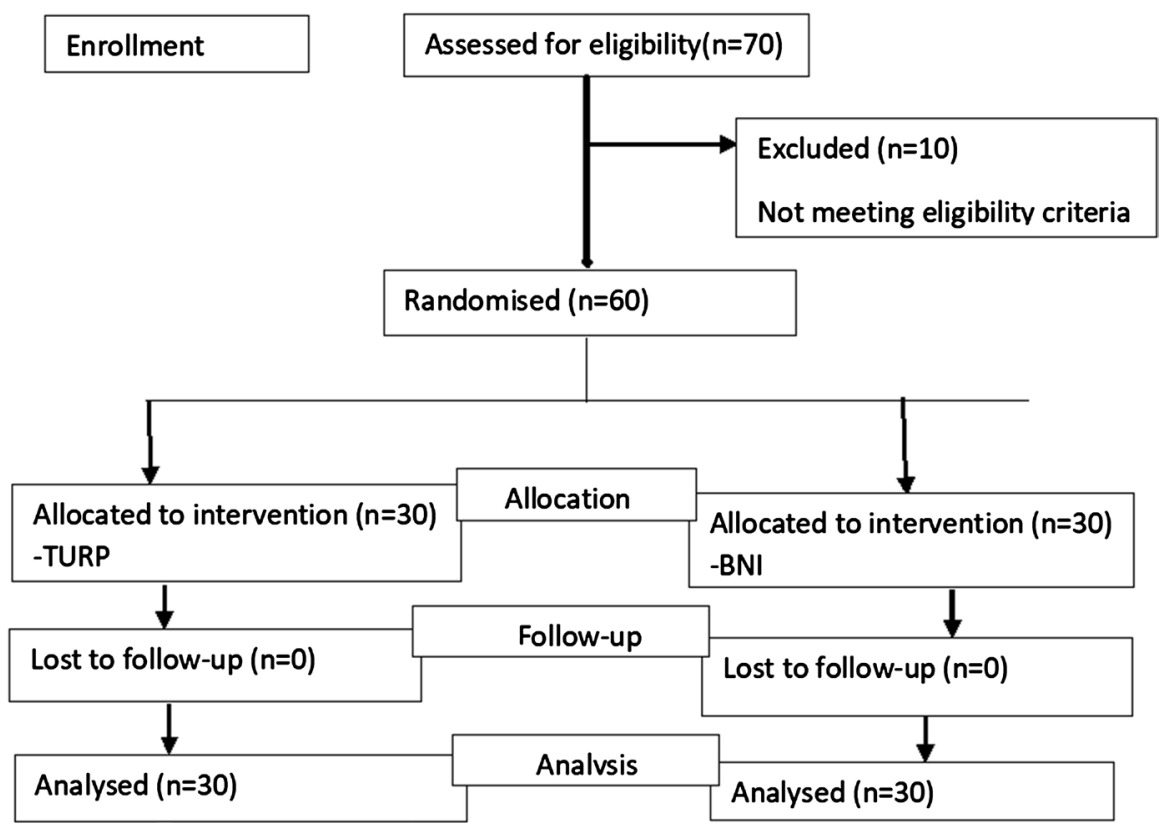

Figure 1. Consort diagram of the study.

\section{Results}

Total 60 patients were included in the study who fulfilled inclusion criteria and there were no lost to follow up cases. This was a randomized controlled study where the patients were divided into two groups, one group underwent BNI and the other underwent TURP. There were 30 patients in each group. Pre-operative data of both groups are compared in Table 1 .

Table 1. Pre-operative characteristics of TURP and BNI group (student t-test was used).

\begin{tabular}{cccc}
\hline Pre-operative variable & TURP Group $(\mathrm{n}=30)$ & BNI Group $(\mathrm{n}=30)$ & p value \\
\hline Age (mean \pm SD) in years & $66 \pm 2.94$ & $66.10 \pm 7.45$ & 0.969 \\
Prostate gland size(g) & 27.10 & 24.70 & 0.307 \\
Mean (range) & $(14-30)$ & $(14-30)$ & 0.881 \\
Symptom score $($ IPSS) & $18.70 \pm 2.95$ & $18.90 \pm 2.96$ & 0.324 \\
Peak flow rate $\left(\mathrm{Q}_{\max }\right)$ in $\mathrm{ml} / \mathrm{s}$ & $6.35 \pm 4.49$ & $4.51 \pm 3.57$ &
\end{tabular}

Table 1 shows that there is no statistical significant difference in pre-operative variables in TURP and BNI groups and the randomisation had generated a well-matched group for the study. This indicates the there was no bias in the participant selection process.

Table 2 shows that total symptom score (IPSS) after both TURP and BNI have reduced significantly $(\mathrm{p}<0.05)$ but the comparison of percentage change between the groups is not significant $(\mathrm{p}>0.05)$. Maximum flow rates also have 
improved significantly after both procedures $(\mathrm{p}<0.05)$ and interestingly there was a statistical significant difference in the percentage change from pre-operative status of peak flow rate in BNI group $(207.91 \% \pm 153.89 \%$ in BNI group compared to $95.25 \% \pm 40.99 \%$ in TURP group) as evidenced by $\mathrm{p}$ value of 0.048 . These results indicate that TURP and BNI, both are equally effective in relieving symptoms and improving urinary flow rates (BNI having an upper hand in the later).

Table 2. Comparison of different variables between TURP and BNI group Pre and 4 months post operatively ( $t$ test for equality of means was applied).

\begin{tabular}{cccc}
\hline Variables & TURP $(\mathrm{n}=30)$ & BNI $(\mathrm{n}=30)$ & p value \\
\hline Pre-op IPSS score & 18.70 & 18.90 & 0.881 \\
Post op IPSS score & $5.70 \pm 1.16$ & $6.00 \pm 1.41$ & 0.704 \\
Pre -op peak flow rate (ml/s) & 6.35 & 4.51 & 0.324 \\
Post op peak flow rate $(\mathrm{ml} / \mathrm{s})$ & $16.41 \pm 2.28$ & $15.95 \pm 2.58$ & 0.048 \\
\hline
\end{tabular}

Table 3 shows that operating time, blood loss post-operative hospital stay are less in BNI group than that of TURP group and the statistically high difference was seen as noted by $\mathrm{p}$ value $<0.001$.

Table 3. Comparison of operative and post-operative parameters. (students $t$ test was applied).

\begin{tabular}{cccc}
\hline Variable & TURP $(\mathrm{n}=30)$ & BNI $(\mathrm{n}=30)$ & p value \\
\hline Duration of surgery (in min) & $32.80 \pm 8.94$ & $12.00 \pm 3.56$ & $<0.001$ \\
Post op fall in Hb & $1.09 \pm 0.32$ & $0.47 \pm 0.17$ & $<0.001$ \\
Duration of post op catheter removal (in days) & 1.9 & 1.5 & 0.141 \\
Post op hospital stay (in days) & $2.70 \pm 0.48$ & $1.50 \pm 0.53$ & $<0.001$ \\
\hline
\end{tabular}

\section{Discussion}

Transurethral resection of the prostate (TURP) and Bladder neck incision (BNI) both are accepted treatment for small size prostate causing obstruction. TURP has for many years been the most commonly performed transurethral operative procedure for resection of obstructing prostatic adenoma [8]. This randomised controlled study compares the subjective and objective improvement resulting from BNI with that of TURP. Statistical analysis was done on collected data and found that there was no statistically significant difference in subjective and objective parameters in between two groups preoperatively.

There is no clear-cut definition for small size gland ( $20 \mathrm{~g}$ by Orandi, $30 \mathrm{~g}$ by Yang et al., up to $50 \mathrm{~g}$ by Aliaga et al. [9]) so we have taken 30 grams as upper limit of gland size as most of the studies in past have compared efficacy of BNI in prostate size 30 grams. Also too large a prostate than 30 grams may not apparently respond to BNI only. There is no direct correlation between the size of the gland and BOO symptomatology as small glands may be more symptomatic 
than hugely enlarged prostate. Mebust et al. [10] in a review of 3885 TURPs, reported an average resected prostatic weight of $22 \mathrm{~g}$ of tissue. In $65 \%$ of the TURPs less than $20 \mathrm{~g}$ was resected. According to these data, most BEP surgically treated cases would be eligible for BNI if the procedure was aimed at patients with estimated resected prostatic weight of less than $20 \mathrm{~g}$. as demonstrated in this article, the increased use of BNI as opposed to TURP is clinically indicated, and BNI's potential to reduce the health service costs is substantial.

We compared intraoperative variable procedure time which had shown statistically significant advantage for BNI (12 min vs $32.8 \mathrm{~min}$ ). This outcome correlated with all the reference studies. The duration of surgery in each case was calculated from the time of starting of resection in TURP and from the time of start of incision in BNI to the time till start of insertion of Foley's catheter. In Riehmann et al. [8] series it was $55 \mathrm{~min}$ and $23 \mathrm{~min}$; in Jahnson et al. series it was $32 \mathrm{~min}$ and $15 \mathrm{~min}$; in Soonawalla et al. [11] series it was 59.2 and $20.4 \mathrm{~min}$ respectively for TURP and BNI. The operating time was directly related with the amount of irrigation fluid used, risk of TUR syndrome development and partly with blood loss.

There was significant improvement of symptoms in both the groups but both groups were found to be equally efficacious as difference in IPSS score and Qmax improvement was statistically significant amongst two groups. Compared to baseline/pre-op, the post-operative IPSS were lower statistically at all follow up visits after both TURP and BNI. Mean improvement in TURP group was 8 , 12.8 and 13.0 after 1 week, 1 month and 4 months of surgery respectively and the mean improvement in BNI group was 7.9, 12.7 and 12.9 respectively which were comparable with no statistical significance. These indicate that both the procedures are equally effective in reducing symptom score. These confirm the experience of Lourenco et al. [12] (2010) Jahnson et al. (1998) [4], Riehmann et al. [8] (1995) who found no significant difference in symptom score improvement between the groups.

In our study, pre-operative mean $\mathrm{Q}_{\max }$ were $6.35 \mathrm{ml} / \mathrm{s}$ and $4.51 \mathrm{ml} / \mathrm{s} \mathrm{respec}$ tively in TURP and BNI group. There were significant improvements $(\mathrm{p}<0.001)$ in flow rates in both TURP and BNI group post operatively as shown by the post-op $\mathrm{Q}_{\max }$ values $-14.27,16.18$ and $16.41 \mathrm{ml} / \mathrm{s}$ and $13.64,15.52$ and $15.95 \mathrm{ml} / \mathrm{s}$ respectively at 1 week, 1 month and 4 months post-operatively. There is insignificant difference between the two groups $(\mathrm{p}>0.1)$. these results agree with those of Christensen et al. [13] (1990), Riehmann et al. [8] (1995). Dorflinger et al. [14] (1987) found the change from $10.1 \mathrm{ml} / \mathrm{s}$ and $9.2 \mathrm{ml} / \mathrm{s}$ pre-operatively to $15 \mathrm{ml} / \mathrm{s}$ and $19 \mathrm{ml} / \mathrm{s}$ post-operatively in TURP and BNI group respectively. In Larsen et al. (1987) series these changes were $7.4 \mathrm{ml} / \mathrm{s}$ and $8.6 \mathrm{ml} / \mathrm{s}$ to $18.5 \mathrm{ml} / \mathrm{s}$ and 20.6 $\mathrm{ml} / \mathrm{s}$; however one study showed significant difference between the groups (Jahnsen et al. [4]). They postulated that the removal of gland resulted in creation of a good channel in TURP group. The mean difference between preop and postop $\mathrm{Q}_{\max }$ (improvement) in TURP group was 10.06 and BNI group was 11.54 
which shows no statistical significance and correlated with the reference studies.

The mean improvement in $\mathrm{Q}_{\max }$ at 1 week of TURP was 14.27 as compared to 13.64 after BNI. The percentage improvement of the same parameters was 67.71 \pm 30.09 with TURP and $148.91 \pm 99.09$ in BNI group, the $p$ value of which is 0.025 , statistically significant. So, there was significantly much more increase in $\mathrm{Q}_{\max }$ in BNI group. The mean percentage improvement at 1 month of TURP and BNI were comparable and not statistically significant. The mean percentage improvement in $\mathrm{Q}_{\max }$ at 4 months of TURP was $95.25 \pm 40.99$ and $207.91 \pm 153.89$ with BNI. $\mathrm{p}$ value of which is 0.048 , proving it to be statistically significant. There was much more improvement in $\mathrm{Q}_{\max }$ in BNI group at 4 months in comparison to TURP group.

IN TURP arm, all the patients were able to pass urine and none of them required recatheterisation. In BNI group, 1 patient did not void after decatheterisation. He was recatheterised but was having clot retention which didn't clear even on irrigation. Clot evacuation was done for him and Foley's was removed the next day and patient could void clear urine. One patient in BNI group had urinary incontinence after the decatheterisation. He was managed medically by anticholinergic therapy and pelvic floor exercises (Kegel's exercises). He was on Foley's pre-operatively for long time which might be the reason for his incontinence. Three patients in both the groups had symptoms of urgency which was managed by anticholinergic therapy.

Jahnson et al. recommended delayed decatheterisation in BNI group due to risk of adhesion between lateral lobes. In our study 10 patient in TURP group and 20 in BNI group were decatheterised on post-operative day 1 and 15 patients in TURP group and 10 patients of BNI group were decatheterised on post-operative day 2 and watched for retention. The mean duration of post-operative catheterisation was 1.9 days for TURP group and 1.5 days for BNI group. In Golam Robbani et al. series it was 3.86 days for TURP and 2.13 days for BNI group. In Riehmann et al. (1995) series it was 2.5 days for TURP and 1.4 days for TUIP. Soonawalla et al. (1992) found 3.01 days (mean) and 2.62 days (mean) postoperative catheterization required for TURP and TUIP group respectively. Dorflinger et al. (1992) series show 2 days (median) with postoperative catheterization in both the groups. Duration of postoperative catheterization in this series is comparable to other series.

Mean post-operative hospital stay in our study was 2.70 days and 1.50 days for TURP and BNI respectively. The difference was statistically significant ( $\mathrm{p}<$ 0.001) and is in favour of BNI. In Soonawalla et al. [11] (1992) series the mean post-operative hospital stay was 7.16 days and 6.03 days respectively in TURP and BNI groups. In Riehmann et al. (1992) series it was 4.3 and 3 days respectively for TURP and BNI groups.in a meta-analysis by Yang et al. [3] (2001) the mean hospital stay was 4.4 to 8.4 and 4.4 to 6.2 days respectively for TURP and BNI and the pooled difference was marginally statistically and clinically significant.

Blood loss was calculated by comparing the pre-operative haemoglobin (sample taken on the morning of surgery) and post-operative haemoglobin (sample 
taken on POD1). The mean of post-operative fall in haemoglobin after TURP was $1.09 \pm 0.32$ and $0.47 \pm 0.17$ in BNI group. This result was statistically significant as shown by a $\mathrm{p}$ value $<0.001$. There is significantly less bleeding in BNI group because of minimal dissection. None of the patient required blood transfusion. Soonawalla et al. [11] (1992) series shows that 38 patients (34.5\%) out of 110 in TURP group and no patient (0\%) in TUIP group required blood transfusion. Jahnson et al. [4] (1998) series required per-operative transfusion in one patient out of 42 in TURP group and no patient out of 43 in TUIP group.

To be an attractive alternative to TURP, BNI must have other advantages, apart from being able to relieve bladder outlet obstruction. Many reports comparing transurethral incision to transurethral resection of prostate have documented decreased operative time, irrigation fluid requirement, blood loss and requirement of blood transfusion, post-operative catheterisation period, and hospital stay with BNI.

The treatment of BEP is aimed at total regression or satisfactory alleviation of symptoms, which results in improvement of quality of life, allowing adequate emptying of bladder freely and under low pressure [15].

The limitation of our study was that of a small sample size and large-scale, multicentric randomized controlled trial is now required to evaluate comprehensively the effectiveness, impact on quality of life and overall cost of transurethral prostatic incision compared with transurethral prostatic resection.

\section{Conclusions}

In our study, outcome assessed by comparing difference (improvement) in variables which shows BNI is equally efficacious to TURP in small size BEP (less than $30 \mathrm{~g}$ ). Intra operative variable shows significant reduction in operative time. The post-operative hospital stay after BNI was significantly shorter than after TURP and this finding is consistent with the results from other studies. There was much more improvement in $\mathrm{Q}_{\max }$ in BNI group at 4 months in comparison to TURP group. BNI is also applicable in patients in urinary retention, has a shorter operative time, and can be performed under local anesthesia. In the present study, the time of indwelling transurethral catheter after TUIP was significantly shorter than after TURP and this may minimize the risk of urinary tract infection.

TUIP can be a better choice in selected group of patients like those not fit for prolonged anesthesia due to age or those who will not withstand hemodynamic instability caused by fluid overload on irrigation during lengthy procedure like TURP.

\section{Conflicts of Interest}

The authors declare no conflicts of interest regarding the publication of this paper.

\section{References}

[1] McConnell, J.D., Barry, M.J. and Bruskewitz, R.C. (1994) Benign Prostatic Hyper- 
plasia: Diagnosis and Treatment. Agency for Health Care Policy and Research. Clinical Practice Guideline: Quick Reference Guide, 8, 1-17.

[2] Madersbacher, S., Djavan, B. and Marberger, M. (1998) Minimally Invasive Treatment for Benign Prostatic Hyperplasia. Current Opinion in Urology, 8, 17-26.

https://doi.org/10.1097/00042307-199801000-00004

[3] Yang, Q., Peters, T.J., Donovan, J.L., Wilt, T.J. and Abrahams, P. (2001) Transurethral Incision Compared with Transurethral Resection of the Prostate for Bladder Outlet Obstruction: A Systemic Review and Meta-Analysis of Randomised Controlled Trials. Journal of Urology, 165, 1526-1532. https://doi.org/10.1016/S0022-5347(05)66342-2

[4] Jahnson, S., Dalen, M., Gustavsson, G. and Pedersen, J. (1998) Transurethral Incision versus Resection of the Prostate for Small to Medium Benign Prostatic Hyperplasia. British Journal of Urology, 81, 276-281.

https://doi.org/10.1046/j.1464-410X.1998.00535.x

[5] Yang, Q., Abrams, P., Donovan, J., Mulligan, S. and Williams, G. (1999) Transurethral Resection or Incision of the Prostate and Other Therapies: A Survey of Treatments for Benign Prostatic Obstruction in the UK. BJU International, 84, 640-645.

https://doi.org/10.1046/j.1464-410x.1999.00276.x

[6] Golam Robbani, A.B.M., Salam, M.A. and Anowarul Islam, A.K.M. (2006) Transurethral Resection (TURP) versus Transurethral Incision (TUIP) of the Prostate for Small Sized Benign Prostatic Hyperplasia: A Prospective Randomized Study. TAJ: Journal of Teachers Association, 19, 50-56. https://doi.org/10.3329/taj.v19i2.3149

[7] National Clinical Guideline Centre (UK) (2010) The Management of Lower Urinary Tract Symptoms in Men (Internet). (NICE Clinical Guidelines, No. 97.) Appendix $\mathrm{H}$, International Prostate Symptom Score (IPSS). Royal College of Physicians, London, UK.

[8] Riehmann, M., Knes, J.M., Heisey, D., Madsen, P.O. and Bruskewitz, R.C. (1995) Transurethral Resection versus Incision of the Prostate: A Randomized, Prospective Study. Urology, 45, 768-775. https://doi.org/10.1016/S0090-4295(99)80081-8

[9] Rodrigo Aliaga, M., Valls Blasco, F. and Jimenez Cruz, J.F. (1998) Lasers as an Alternative to the Endoscopic Surgery in BPH. Actas Urológicas Españolas, 22, 17-22.

[10] Mebust, W.K. (1988) Surgical Management of Benign Prostatic Obstruction. Urology, 23, 12-15.

[11] Soonawalla, P.F. and Pardanani, D.S. (1992) Transurethral Incision versus Transurethral Resection of the Prostate. A Subject and Objective Analysis. British Journal of Urology, 70, 174-177. https://doi.org/10.1111/j.1464-410X.1992.tb15698.x

[12] Lourenco, T., Shaw, M., Fraser, C., MacLennan, G., N’Dow, J. and Pickard, R. (2010) The Clinical Effectiveness of Transurethral Incision of the Prostate: A Systematic Review of Randomised Controlled Trials. World Journal of Urology, 28, 23-32. https://doi.org/10.1007/s00345-009-0496-8

[13] Christensen, M.M., Aagaard, J. and Madsen, P.O. (1990) Transurethral Resection versus Transurethral Incision of Prostate. A Prospective Randomised Study. Urologic Clinics of North America, 17, 621-630.

[14] Dorflinger, T., Jensen, F.S., Krarup, T. and Walter, S. (1992) Transurethral Prostatectomy Compared with Incision of the Prostate in the Treatment of Prostatism Caused by Small Benign Prostate Glands. Scandinavian Journal of Urology and Nephrology, 26, 333-338. https://doi.org/10.3109/00365599209181222 
[15] Tkocz, M. and Prajsner, A. (2002) Comparison of Long-Term Results of Transurethral Incision of the Prostate with Transurethral Resection of the Prostate, in Patients with Benign Prostatic Hypertrophy. Neurology and Urodynamics, 21, 112-116. https://doi.org/10.1002/nau.10013 